\title{
CONVERGENCE IN MEASURE FOR SEMIGROUP-VALUED INTEGRALS
}

\author{
ISIDORE FLEISCHER
}

\begin{abstract}
The semigroup-valued integral of $\mathbf{M}$. Sion $[\mathbf{S}]$ is reformulated for a general notion of approximation by sums of values taken by a set function integrand. A convergence in measure theorem is established, which yields both his pointwise dominated convergence theorem as well as an integrability criterion which specializes to his existence theorem.
\end{abstract}

In [S] M. Sion introduced and developed an "integral process" for set functions with values in a uniform semigroup, and more particularly for (possibly multi-) uniform space-valued point functions with respect to a topological semigroup-valued finitely additive set function, on the product of whose image with that of the point function a suitable uniform semigroup-valued "multiplication" is available. Among the developments is a convergence theorem for sequences of integrable point functions, patterned after Lebesgue's, thus postulating almost everywhere convergence. Classically this type of convergence theorem can be deduced from one with the weaker hypothesis of convergence in measure; the deduction of this hypothesis from almost everywhere convergence will also be available here-although the usual route via Egoroff's almost uniform convergence seems not be feasible in this generality. This may have been what hindered the development of a convergence in measure theorem in [S]; there may also be some confusion connected with the asserted equivalence of the formulations on pages 40 and 41 (whose verification by "straightforward checking" this reader was unable to believe and conjectures to be incorrect).

The task of setting up the indicated convergence in measure theorem is facilitated by the availability of the preceding [F], which generalized the Banach space-valued result from [DS] to a non-absolutely integrable setting ${ }^{1}$ and was liberally sprinkled with hints as to the modifications which would make it applicable to uniform spaces. The only essentially new ingredient which has to be provided is the displacement of the directed order, which governed convergence to the integral, from the set of finite disjoint subfamilies in the domain of the measure, to an auxiliary set: i.e. the replacement of the order on these subfamilies by a net on them. The result achieved

Received by the editors March 10, 1986.

1980 Mathematics Subject Classification (1985 Revision). Primary 28B10.

${ }^{1}$ This had actually been done already in [B] 
not only weakens the hypothesis of pointwise convergence to convergence in measure, but also provides conditions for assuring (rather than postulating) the integrability of the limit function; it thus encompasses both the existence and the limit under the integral sign results in $[\mathbf{S}]$.

The organization of the sequel is as follows: A general integral for multi-semigroup-valued set functions is first set up, by equipping the finite disjoint subfamilies of their domains with nets governing the convergence of approximating sums, subject to natural axioms to assure additivity of the integral in integrand and integration domain. A rather rudimentary convergence criterion is formulated in this setting, which contains the kernel of the later existence and limit interchange results. The set functions are then specialized to "products" of uniform space-valued point functions by set functions with values which map their range to the semigroup. There follows a topological definition of "convergence in measure on a set of bounded variation to a bounded point function", for which the criterion furnishes convergence of the integrals to that of the limit function (the full result requiring as usual supplementary absolute continuity) _ in particular an integrability criterion for such functions which generalizes to this context the existence theorem of [S]. Finally, the pointwise convergence theorem of $[\mathbf{S}]$ is shown to follow from the full convergence in measure theorem.

The integrals will be defined over (some) subsets of a basic set $S$ and take values in a uniform semigroup $Z$ : i.e. in a uniform space with a commutative jointly uniformly continuous addition [S, p. 2]. The integrands will be defined on a class $H$ of subsets of $S$ and take (possibly multi-) values in $Z$; by means of the addition in $Z$ they may be extended additively to the class $\Sigma$ of finite disjoint subfamilies of $H$ (so as to send union performable in $\Sigma$ to (subset) sum in $Z$ ); and the integrals are to be limits in $Z$ of nets of such sums. These defining nets will be specified via nets in $\Sigma$ : an integral over a "domain of integration" $A \subset S$ will be specified by a map from a directed set $D(A)$ to $\Sigma$, and the integral over $A$ of a $Z$-valued function $\tau$ on $H$ will be the limit of the net obtained by composing this map with the additive extension of $\tau$ to $\Sigma$. For the purpose at hand, these defining nets $I$ from the $D(A)$ to $\Sigma$ should satisfy: for any integration domain $A, I$ restricted to $D(A)$ should take as values disjoint subfamilies made up only of subsets $(\in H)$ of $A$; over unions of disjoint such $A$ 's $I$ should operate "disjointly", i.e. should send elements of $D\left(A \cup A^{\prime}\right)$, which (at least finally) is to equal (isomorphically) $D(A) \times D\left(A^{\prime}\right)$, to the (still pairwise disjoint) union of the respective values of $I$ on their components. ${ }^{2}$ These conditions are met both by the refinement order of [F, top of p. 370]-where the domains of integration were the elements of a ring of subsets whose finite disjoint families, directed by refinement and increasing union and contained in a particular domain $A$, constituted a $D(A)$ for an identity net; and by the integral process of [S, pp. 16, 17] - at least for domains whose disjoint countable covers by sets in $H$ are

\footnotetext{
¿To an infinite disjoint union of $A$ 's which is an integration domain, $D$ should assign the union of all finite products of the $D(A)$ 's, ordered so that an element is dominated by another with possibly additional components, provided it is dominated on each of its components.
} 
refined by partitions (these would include the sets in an intersection closed $H$ ); the integral over an $A$ is defined to be the limit of the integrand along the "iterated filterbase" $\left[\mathbf{F}^{\prime}\right]$ of unconditional summation on finite parts of the partition indexed by refinement of partitions: in the formalism of [S, p. 17], the $D(A)$ are pairs from which $I$ may be produced by evaluating the second component at the first.

Continuity of addition in $Z$ now entails that if $\tau$ is integrable over each of disjoint $A, A^{\prime}, \ldots$, it will be over their union, with value the sum of the integrals ${ }^{3}$. A converse holds for $Z$ a (complete) group: from the integrability over a disjoint union of integration domains will follow integrability over each of them (cf. the proof of [S, 5.3, p. 32]). In addition, the integral is additive in the integrand in the same strong sense: that the integrability of a sum follows from that of the summands.

In this setting one may establish (easily - the proof may safely be omitted): Sufficient that a multi-valued function $\tau$ on $H$ to a complete $Z$ be integrable over $S$ is: that for every entourage $W$ of $Z$ there exist an integration domain $A$ some function $\sigma$, integrable over which, approximates $\tau$ finally in $D(A)$ to closeness $W$-i.e. every element of $\tau \pi$ is within $W$ of some element of $\sigma \pi$, for the $\pi$ in the image by $I$ of some final subset of $D(A)$; as well as the values of $\tau$ being finally in $S$, within $W$ of every set of its values final in this $A$-i.e. the $W$-neighborhood of the image by $\tau \circ I$ of each final subset of $D(A)$ containing the $\tau \circ I$ image of some final subset of $D(S)$. Moreover if a choice of such functions $\sigma$ and sets $A$ assigned to the $W$ is construed as a net of pairs indexed by the (opposite-to-inclusion directed) entourage system of $Z$, then $\int_{A} \sigma$ converges to $\int_{S} \tau$; and so at last if also $\left(\int_{A} \sigma, \int_{S} \sigma\right)$ $\in W$ for such a choice, then $\int_{S} \tau$ is the limit of $\int_{S} \sigma$.

Given that $\sigma \pi$ converges in $Z$ along $D(A)$, the first requirement entails that every element of $\tau \pi$ is close to every element of $\sigma \pi$ finally in $D(A)$ i.e. that $(\tau \pi, \sigma \pi)$ sends a final subset into $W$ in $Z \times Z$; and then the second entails that $(\tau \bar{\pi}, \tau \pi)$ sends the product, of a final subset of $D(S)$ by one final in $D(A)$, into $W$. This leads to a more flexible way of formulating the criterion: There should exist a net of integration domains $A$ each of which has assigned a function $\sigma$ integrable over it; the $A$ 's having also assigned a net of $\pi$ 's, one can form their "iterated" $\left[F^{\prime}\right]$ section filterbase ${ }^{4}$. The first requirement then comes to the image in $Z \times Z$ of this filterbase by $(\tau \pi, \sigma \pi)$ refining the uniformity's entourage filter; the second to the image by $(\tau \bar{\pi}, \tau \pi)$ of the product, sections of $D(S)$ by this iterated filterbase, refining the entourage filter; and the last, when the $\sigma$ are also integrable over $S$, to the final (along the net) pairs $\left(\int_{A} \sigma, \int_{S} \sigma\right)$ refining it.

When the complement $A^{\prime}$ of $A$ is also an integration domain then, since the values of $\tau$ in $S$ are finally sums of such in $A$ and in $A^{\prime}$, the requirement of their closeness to final values in $A$ would be met by the $\tau \pi^{\prime}$, finally on $D\left(A^{\prime}\right)$, translating by

\footnotetext{
${ }^{3}$ in the setting of $[\mathbf{S}]$, also for countably many disjoint $A$ 's over the union of which $\tau$ is integrable (cf. [S. 4.2, p. 18]). The integrals over the $A$ 's sum unconditionally-i.e. cofinitely - to that over the union.

${ }^{4}$ i.c. the filterbase, on the disjoint union of the $D(A)$, of all unions of final sets indexed by a final set of $A$ 's.
} 
addition the $\tau \pi$, on each final subset of $D(A)$, so as to keep image uniformly close to argument; similarly, the last condition from $\int_{A^{\prime}} \sigma$ existing and translating $\int_{A} \sigma$ not further away than $W$; also the first, of final approximation of $\tau$ by $\sigma$ in $D(A)$, from one of $\sigma, \tau$ being an appropriate sum of the other with a function whose values on a final subset of $D(A)$ have close additive translations. When $Z$ has a zero, closeness of the additive translate, uniformly in the translated argument, is ensured by closeness of the translating element to zero, by virtue of uniform continuity of addition. For $Z$ a group, the first condition follows from the difference set function $\sigma-\tau$ sending a final subset in $A$ into a neighborhood $W$ of 0 , the second from $\tau$ so sending a final subset in $A^{\prime}$, while the last comes to $\int_{A^{\prime}} \sigma \in W$. The result is then recognizable as a variant of the Satz [F, p. 368] (of its first part, since the integral there is defined by an iterated rather than a single limit) which allows the domain of definition to vary with the set function but specifies the limiting process by a directed order imposed directly on the finite disjoint families rather than by the present more general device of a net.

Next, specialize the $Z$-valued functions $\tau$ (not as far) as in [S, Chapter II] to "products" of point functions on $S$, taking (possibly multi-) values in a uniform space $X$, with a set function $\lambda$ on $H$ taking values in a space $Y$ of uniformly continuous maps from $X$ to $Z$. By composing with the uniformly continuous addition in $Z$, specifically with the map from $Z^{n}$ to $Z$ which sends every $n$-tuple to its sum, the elements of $Y^{n}$ become uniformly continuous maps to $Z$ from $X^{n}$; since the latter is equipped with the product uniformity, it becomes possible to compare the "modulus" of uniform continuity for maps in different $Y^{n}$ : a subset of $\cup Y^{n}$ is taken to be "equi-uniformly continuous" when, for every entourage $W$ of $Z$, there exists an entourage $U$ of $X$ such that (for every $n$ ) the part of the subset in $Y^{n}$ sends $U^{n}$ into $W$. One can then assign a subset $B$ of $S$ "bounded (semi) variation" of $\lambda$ just when the continuity of $\lambda \pi$ is equi-uniform over all the finite disjoint $\pi$ in $B$, and "final bounded variation" when it is equi-uniform over all subfamilies of the $\pi$ in the image of some final subset of $D(B)$. Note that a "bound" on $B$ for the variation (construed as the assignment to each $W$ of a $U$ whose finite powers are sent into $W$ by all these $\lambda \pi$ ) is also a bound on every subset of $B$; and a final bound also one on each $A \subset B$ which is, with $B-A$, an integration subdomain: thus if $\lambda$ is of (final) bounded variation on $B$, it will have a common (final) bound on all subsets (occurring in a partition into integration subdomains) of $B$.

The (possibly multi-valued) point functions extend so as to send the subsets in $H$ to subsets of $X$ and the functions in $Y$ from $X$ to $Z$ extend to send subsets of $X$ to subsets of $Z$ : one can thus evaluate the "product" of $t$ and $\lambda$ on an $A$ (as the image of $t A$ by $\lambda A$ ) to obtain a subset of $Z$; add these subsets to evaluate this product on any $\pi$; and take the limit of such sums along $D(A)$ for $\int_{A} t \lambda$.

Consider the above convergence criterion over a subset $B$ of final bounded variation: the existence of a net of point functions $s$ "converging" to $t$-in the sense that for each $s$ there is an integration subdomain $A$ of $B$, over which $s$ is integrable with respect to $\lambda$ and at each of whose elements (every value of) $t$ is so close to (some value of) $s$ that the sets of these pairs refine the entourage filter of $X$ along the net - assures the final approximability in $D(A)$ of $t \lambda$ by $s \lambda$. That of $t \lambda$ on $B$ by 
$t \lambda$ on $A$ would follow if the multi- $Z$-valued set function $t(B) \lambda$ sent the $\pi^{\prime}$ in $A^{\prime}$ (a final subset in, when $A^{\prime}$ is an integration domain), the complement in $B$ of the subdomain $A$ on which $s$ approximates $t$, to a subset of $Z$ the additive translations by whose elements have graphs whose unions refine the entourage filter along the net (e.g. to a subset of $Z$ which converges along the net to an existent 0 ). Refinement of the entourage filter by the graphs of translation by the images of $\pi^{\prime}$ in (a final subset of) the $A^{\prime}$, may be formulated function theoretically as convergence of the translations to the identity function, uniformly over $Z$, along the net of the $A^{\prime}$ (the iterated filterbase in the $A^{\prime}$ indexed by this net). When $\int_{A^{\prime}} t \lambda$ exists, this entails (comes to) that its graph of translation refines the entourage filter along the net, convergence of $\int_{A^{\prime}} t \lambda$ to 0 again sufficing by uniform continuity of addition. Similarly, the last condition (with the $s$ integrable over $B$ )-refinement by $\left(\int_{A} s \lambda, \int_{B} s \lambda\right)$ along the net-would follow from a kind of "absolute continuity" of the net of $s \lambda$. It would again come to requiring the $s \lambda$ to "be small" (on some final subset) in $A^{\prime}$ far along the net. With a single such $A^{\prime}$ required to be available for $s$ final in the net, on whose complement also $t$ is approximated uniformly by $s$ final in the net, this is the "quasi-uniform" convergence of [Mi, 1.6.1]; the convergence theorem [Mi, 1.8.1] for this type of convergence on an $S$ of bounded variation (" $v$ is bounded" 1.3.4) is a special case.

For $t$ to be integrable over all of $S$, it would suffice that it be integrable over the complement of one of these $B$ 's; to have in addition its integral the limit of that of the $s$ over $S$, one should be able to choose for each $W$ such a $B$ in which the approximating $\int_{A} s$ were cofinally within $W$ of their integrals over $S$ and that their limit, along their net, were within $W$ of $\int_{S} t$-i.e. to find a net of such $B$ 's, each having assigned a net of $s$-indexed subdomains $A$ over which the integrals of $s$ converge to $\int_{B} t$, such that the iterated limit is $\int_{S} t$ (say because $\int_{S-B} t$ exists and converges to zero) and such that for each $s\left(\int_{A} s, \int_{S} s\right)$ along the net of $B$ 's meets the entourage filter (say because $\int_{S-A} s$ clusters at zero).

From the convergence theorem can be extracted an integrability criterion. This is accomplished by construing the $\tau \pi$, which it is desired to have converge, as values of the linear extension of $\lambda$ to "simple" functions (cf. the treatment in [DS] where this is taken as the definition of integrability). Thus the integrability of a function $t$ over a set $B$ of final bounded variation follows from the above when $H$ is a ring, simple functions over whose elements are integrable, and each entourage contains the oscillation of $t$ on the sets of some finite disjoint $\pi$ (in $B$ ), on whose union's complement (in $B$ ) $t(B) \lambda$ is with in this entourage of 0 .

The smallness of $t(B) \lambda$ on final $\pi^{\prime}$ far along the net of $A^{\prime}$ could be construed as a kind of "convergence in measure to a bounded $t$ " (in $B$ ). Specifically, suppose some of the nets in $U Y^{n}$ are designated to be "convergent". A subset of $X$ would be defined to be "bounded" if every "convergent" net sends it and its $n$-tuples to subsets of $Z$ which have converging graphs of additive translation (or which converge to 0 ): i.e. the subsets of $X$ "bounded" with respect to a "convergence" on $U Y^{n}$ are those which act so as to send $U Y^{n}$ into $Z$ "equicontinuously". Then $t$ "bounded" on $B$ (hence on each of its subsets) and $\lambda$ "converging" in $U Y$ " on (a final subset of, suffices) the $\pi^{\prime}$ in $A^{\prime}$ along the net, assures the approximability of $t \lambda$ 
in $B$ by $t \lambda$ in the $A$ 's. When the "convergence" in $\cup Y^{n}$ and that to 0 in $Z$ are given by (convergence to 0 of) nonnegative norms, a subset of $X$ for which $\left|\sum x_{i} y_{i}\right| /\left|\left\langle y_{i}\right\rangle\right|$ remains bounded (on all of $\cup Y^{n}$ and all $n$-tuples from the subset) is "bounded" for this "convergence"; more particularly, if $X$ itself is so normed that $\left|\sum x_{i} y_{i}\right| \leqslant \max \left|x_{i}\right|$ - $\left|\left\langle y_{i}\right\rangle\right|$, then its norm bounded subsets are "bounded". This " $l_{\infty}-l_{1}$ duality" could also be used when the uniformity of $X$ is that of a normed topological group to conclude that $\lambda$ has bounded variation when it sends $\pi$ 's to sets in $\bigcup Y^{n}$ for which $\left|\sum x_{i} y_{i}\right| / \max \left|x_{i}\right|$ is bounded. In [S] this "convergence" on $U Y^{n}$ is obtained, when the function set $Y$ is a commutative monoid under pointwise addition equipped with a compatible topology [S, p. 60], as the inverse image of the neighborhood system at zero under the multi-valued map which sends every $n$-tuple of $y$ 's to the sums in $Y$ of (it and) all its subtuples. The composition of this map with $\lambda$ as extended to map the $\pi$ 's to $\cup Y^{n}$, has as image of the $\pi$ (final) in an integration domain $A$, a subset (filterbase) in $Y$ which "measures" $A$-this gives an interpretation of "measure $\lambda$ converging to zero" (in $Y$ ) along an (iterated) filter of $A$ 's. To see that the hypotheses of the "fundamental existence theorem" [S, 4.2, p. 62] are included in the above, note that $[\mathbf{S},(1)$, p. 61$]$ is just the boundedness of $t(S)$ for this convergence on $\cup Y^{\prime \prime}$, while (2)(i) and (ii), finite additivity and $s$-boundedness, entail that "measure $\lambda$ converges to zero" along the cofinite unions of any countable disjoint subfamily of $H$ (use the form of $s$-boundedness [S, (1), top of p. 29] rather than the definition [S, (1), p. 28]). [S, (2)(iii), p. 61] just says that $\lambda$ is of bounded variation on $S$ (which for singleton families yields uniform continuity of $\lambda(H)=Y$ ). Thus if $t$ is "partitionable" [S, p. 44] (i.e. attains arbitrary close oscillation on countable partitions of complements of null sets) one verifies the above hypotheses for the “integral process" order [S, pp. 16,17].

To see that the convergence theorem [S, 5.2, p. 70] is also included, its hypothesis of pointwise convergence on the complement of a null set should be shown to imply convergence in measure. In the classical setting this is accomplished (in the presence of some sort of finiteness) via almost uniform convergence, but although the full conclusion of an Egoroff theorem here would seem to require metrizability of the uniformity on $X$, the usual reasoning actually establishes in measure on the way to almost uniform: see e.g. the proof of 37.1, pp. 199, 200 in [Mu]. The argument only depends on having the measure converge to 0 on sequences of $H$ decreasing to null sets-whose analogue is available here, by disjointifying the sequence and applying finite additivity and $s$-boundedness of $\lambda$ (as in the preceding paragraph): such decreasing sequences have terms with arbitrarily small "measure". Now to find sets at whose elements one of the almost everywhere converging functions is uniformly close to the limit and the "measure" of whose complement is small, use their "partitionability" (more simply than in [S], as indicated in [Mi]'s proof of 1.7.3 for groups): from a partition of common close oscillation of the limit and the $n$th converging function, take the union of those sets which contain a point at which (some values of) these functions are also this close. On the complement of all these sets the $n$th function is almost nowhere so close to the limit, whence the lim sup of these complements over $n$ is (by almost everywhere convergence) a null set, and so one of these complements will have "measure" (will have some set of its final $\pi$ 's 
sent by $\lambda$ into a neighborhood of 0 in $Y$ ) as small as desired. Finally, to obtain the convergence of the integrals over sets not of bounded variation or on which the integrands are not uniformly bounded, the postulated "uniform $s$-integrability" [S, 5.1 , p. 69] provides, in the indexed $A$ 's, subsets of common bounded variation on which the indexing functions have, respectively, a common bound and over whose complements their respective integrals are uniformly small; in view of the finite additivity and $s$-boundedness of $\lambda$, this permits taking the subset indexed by the limit function as the pivotal $B$ in the convergence theorem presented here.

\section{REFERENCES}

[B] R. G. Bartle, A general bilinear vector integral, Studia Math. 15 (1956), 337-352.

[DS] N. Dunford and J. T. Schwartz, Linear operators, Part I, Interscience, New York, 1958.

[F] I. Fleischer, Ein Integralimesaustauschsatz, Math. Ann. 148 (1962), 367-371.

[F'] I. Fleischer, The interchange of order in repeated limits, Rocky Mountain J. Math. 5 (1975), 271-274.

[Mi] H. Millington, Products of group-valued measures, Studia Math. 54 (1975), 7-27.

[Mu] M. E. Munroe, Measure and integration, 2nd ed., Addison-Wesley, Reading, Mass., 1971.

[S] M. Sion, A theory of semigroup valued measures, Lecture Notes in Math., vol. 355, Springer-Verlag, Berlin, 1973.

Department of Mathematics, University of Windsor, 401 Sunset Avenue, Windsor, Ontario N9B 3P4, CANADA 